\title{
Influencia del consumo de sustancias y el deterioro cognitivo en la adherencia al tratamiento antirretroviral en pacientes VIH+
}

\author{
Influence of substance use and cognitive impairment on \\ adherence to antiretroviral therapy in HIV+ patients
}

\author{
Irene Sánchez-Rivero*, Agustín Madoz-Gúrpide**, Carlos Parro-Torres****, \\ Daniel Hernández-Huerta*, Enriqueta Ochoa Mangado****. \\ * Servicio de Psiquiatría. Hospital Universitario Ramón y Cajal, Madrid. Spain. ** Centro de Salud Mental San Blas, Servicio de \\ Psiquiatría, Hospital Universitario Ramón y Cajal (IRYCIS). Universidad de Alcalá, Madrid. Spain. *** Servicio de Psiquiatría. \\ Hospital General Universitario Gregorio Marañón, Madrid. Spain. **** Servicio de Psiquiatría, Hospital Universitario Ramón y \\ Cajal (IRYCIS). Universidad de Alcalá, Madrid. Spain.
}

\section{Resumen}

La adherencia estricta al tratamiento antirretroviral (TAR) es imprescindible para que este sea eficaz en la disminución de la morbimortalidad asociada al VIH. Se ha sugerido que el consumo de sustancias y el deterioro cognitivo constituyen factores de riesgo para una mala adherencia. En este sentido, el objetivo de este estudio es evaluar cuál es la influencia sobre la adherencia al TAR de la disfunción cognitiva, así como del consumo de determinadas sustancias (alcohol, heroína, cocaína, otros estimulantes, cannabis y benzodizepinas) y/o el tratamiento con metadona, en el marco concreto de una población española de referencia. Se realizó un estudio observacional tipo casos y controles con una muestra de 125 pacientes $\mathrm{VIH}+$, que se dividieron en sujetos malos adherentes (casos) y buenos adherentes al TAR (controles). La adherencia se evaluó mediante el reporte de Farmacia Hospitalaria, contrastada con la escala Simplified Medication Adherence Questionnaire (SMAQ) y la opinión del profesional médico de referencia. La función cognitiva fue evaluada con el Test del Mapa del Zoo y el Trail Making Test (TMT), y el consumo de sustancias, mediante un protocolo de historia clínica semi-estructurada. El análisis estadístico se realizó mediante regresión logística binaria. Los resultados mostraron que el abuso de alcohol y el deterioro en la función cognitiva ejecutiva, medida por el Test del Mapa del Zoo, constituyen factores de riesgo independientes para una mala adherencia. No se ha demostrado relación de la adherencia al TAR con el consumo de otras sustancias ni con la puntuación obtenida en el TMT. La detección de deterioro cognitivo mediante el Test del Mapa del Zoo, así como del consumo de alcohol, podrían ayudar a desarrollar estrategias de mejora del cumplimiento terapéutico en pacientes VIH+.

Palabras clave: VIH; Tratamiento antirretroviral; Adherencia terapéutica; Deterioro cognitivo; Consumo de sustancias.

\begin{abstract}
Strict adherence to antiretroviral treatment (ART) is needed to ensure the effectiveness of HIV treatment. The adverse effects of substance abuse and neurocognitive impairment on medication adherence have both been suggested by several studies. Therefore, the aim of this research is to study the relationship among adherence to ART, cognitive dysfunction, and abuse of certain substances (alcohol, heroin, cocaine, other stimulants, cannabis and benzodiazepines) and/or methadone treatment in our social environment. We performed an observational case-control study with a sample of 125 HIV+ patients, who were classified as patients with poor adherence (cases) and subjects with adequate compliance (controls). Adherence was defined by the Hospital Pharmacy and verified with the Simplified Medication Adherence Questionnaire (SMAQ) and the reference physician's clinical impression. Cognitive functioning was measured with the Zoo Map Test and Trail Making Test (TMT). Substance abuse was collected through a semi-structured clinical interview protocol. Statistical analysis was made using a binary logistic regression model. The results indicate that both alcohol abuse and neurocognitive impairment measured by Zoo Map Test were significantly associated with poorer adherence to ART. No significant association was found between adherence and other substance use, or between adherence and TMT score. Screening of cognitive impairment measured by the Zoo Map Test and alcohol abuse may lead to the development of strategies to improve de adherence to ART in HIV+ patients.

Keywords: HIV; Antiretroviral therapy; Medication adherence; Cognitive dysfunction; Substance use.
\end{abstract}

Recibido: Septiembre 2017; Aceptado: Junio 2018.

Enviar correspondencia a:

Irene Sánchez Rivero. Centro de Salud Mental San Blas. Calle Castillo de Uclés 37; 28037 Madrid.

E-mail: irene.sanchez.rivero@gmail.com 


\section{Introducción}

$\mathrm{E}$ 1 Virus de la Inmunodeficiencia Humana (VIH) pertenece a la familia Retroviridae y es causante del Síndrome de la Inmunodeficiencia Adquirida (SIDA), el cual constituye un problema de salud pública de primera magnitud a nivel mundial (Fauci y Lane, 2005). La instauración de regímenes de tratamiento consistentes en la combinación de fármacos antirretrovirales ha permitido que se ralentice la progresión del VIH. Por tanto, el tratamiento antirretroviral (TAR) ha supuesto una importante disminución de la mortalidad y morbilidad asociada al VIH, y, en muchos casos, convierte una infección que antes era invariablemente mortal en una enfermedad crónica (Lovejoy y Suhr, 2009; Tran, Nguyen, Nguyen, Hoang y Hwang, 2013). No obstante, para conseguir una respuesta clínica y virológica completa, y prevenir la aparición de cepas víricas resistentes, es precisa, entre otros factores, una adherencia estricta al tratamiento. La mayoría de autores defienden que para asegurar la eficacia del TAR, la adherencia debe estar por encima del 90-95\%. Sin embargo, mantener una adherencia tan elevada puede resultar difícil para muchos pacientes (Ingersoll, 2004; Lovejoy y Suhr, 2009).

En los últimos años se ha intentado dilucidar qué factores pueden incidir en la adherencia al TAR, con el objetivo de identificar factores de riesgo potencialmente modificables y desarrollar estrategias de mejora de los planes terapéuticos (Thaler, Sayegh, Kim, Castellon y Hinkin, 2015; Tran et al., 2013). Se ha sugerido que pueden estar asociados a un peor cumplimiento el bajo nivel socioeconómico (Peltzer y Pengpid, 2013; Tsuyuki y Surratt, 2015), la presencia de efectos secundarios del tratamiento, la falta de eficacia percibida por los pacientes, el estrés emocional, la ausencia de soporte sociofamiliar, la complejidad de la pauta de tratamiento (Ammassari et al., 2002), la juventud y el deterioro cognitivo (Thaler et al., 2015). Asimismo, se ha propuesto que una peor adherencia se relacione con la presencia de psicopatología, fundamentalmente depresiva (Ammassari et al., 2004); determinados rasgos de personalidad (Hutton y Treisman, 2008), y el consumo de sustancias (Azar et al., 2015). No obstante, existen escasos estudios realizados a este respecto en nuestro contexto sociocultural, por lo que creemos que es necesario investigar las citadas asociaciones en la población española.

El VIH es un virus neurotrópico que invade el sistema nervioso central (SNC) de forma precoz, produciendo neurotoxicidad, neuroinflamación y neurodegeneración. El efecto pernicioso en el SNC puede ocurrir por la acción directa del virus, o bien de forma indirecta a través de la inmunodepresión, la cual puede afectar al SNC en forma de infecciones oportunistas y neoplasias: criptococosis, toxoplasmosis, leucoencefalopatía multifocal progresiva, linfoma cerebral primario, etc. (Bragança y Palha, 2011; Grupo de expertos del Grupo de Estudio de Sida [GeSIDA] y de la Secretaría del Plan Nacional sobre el Sida [SPNS],
2014). Previamente a la introducción del TAR, un gran número de pacientes desarrollaba un deterioro cognitivo grave en la última etapa de la enfermedad, denominado demencia asociada al VIH (HAD, por sus siglas en inglés) por la Asociación Americana de Neurología en 1991. Esta se diferenció de otra entidad en la que no se cumplían criterios de demencia, pero sí existían alteraciones leves que interferían en la vida diaria: alteración cognitiva motora menor (MCMD). En los últimos años, la HAD es cada vez menos frecuente; sin embargo, se ha objetivado que un porcentaje de pacientes de larga evolución presenta deterioro cognitivo, incluso algunos con la infección sistemáticamente bien controlada (Cysique y Brew, 2009; GeSIDA y SPNS, 2014; Solomon y Halkitis, 2008). Con el progreso de la enfermedad, frecuentemente se produce un declive en la función ejecutiva, el funcionamiento motor, la atención, la memoria y la velocidad de procesamiento, y sobrevienen también cambios conductuales, así como apatía y letargia (Anand, Springer, Copenhaver y Altice, 2010; Andrade et al., 2013, Vázquez-Justo, Vergara-Morague, Piñón-Blanco, Guillén-Gestoso y Pérez-García, 2016). Antinori et al. (2007) realizaron una revisión nosológica de las alteraciones cognitivas asociadas al VIH ("criterios de Frascati"), siendo englobadas en el término trastornos neurocognitivos asociados al VIH (HAND). La nueva clasificación propuesta divide esta entidad en tres categorías: alteración cognitiva asintomática (ANI), trastorno cognitivo leve asociado al VIH (MND) y demencia asociada al VIH (HAD). Las dos primeras se caracterizan por el desarrollo de una discapacidad ligera en al menos dos dominios neurocognitivos, sin llegar a cumplir criterios de demencia, y sin interferencia o con interferencia leve, respectivamente, en el funcionamiento habitual del individuo. En la demencia asociada al VIH existe una marcada discapacidad e interferencia en la vida diaria (Antinori et al., 2007; GeSIDA y SPNS, 2014).

En los últimos años, numerosos estudios han expuesto que este deterioro cognitivo es otro de los factores que se relacionan con una peor adherencia al TAR (Andrade et al., 2013; Hinkin et al., 2004; Lovejoy y Suhr, 2009). Un funcionamiento cognitivo mermado puede condicionar la presencia de "olvidos", que es la razón más frecuente para la pérdida de tomas en el tratamiento (Ammassari et al., 2004; Blackstone, Woods, Weber, Grant y Moore, 2013; Hinkin et al., 2002). Otros autores han planteado que es la mala adherencia al TAR la que condiciona una peor evolución cognitiva, ya que acelera la progresión de la enfermedad (Cysique y Brew, 2009; Waldrop-Valverde et al., 2006). No obstante, resulta plausible que se trate en realidad de una relación bidireccional (Andrade et al., 2013; Hinkin et al., 2004; Lovejoy y Suhr, 2009). Hay que tener en cuenta, además, que el TAR habitualmente supone un régimen de tratamiento complejo, que implica mecanismos relacionados con la planificación y la toma de decisiones, y por tanto requieren una notable demanda cognitiva. En tal caso, el 
déficit cognitivo ha de tenerse en cuenta en el diseño del tratamiento, con el objetivo de establecer un régimen apropiado para las características de cada paciente (Blackstone et al., 2013; Hinkin et al., 2002; Solomon y Halkitis, 2008). No existe uniformidad en la literatura respecto a qué áreas cognitivas influyen más en la adherencia al TAR. Según la evidencia disponible, una peor adherencia podría asociarse a déficits en las funciones ejecutivas, la capacidad de aprendizaje, la atención, la memoria de trabajo, la memoria prospectiva y la velocidad de procesamiento. No obstante, los resultados son heterogéneos entre los distintos estudios y los mecanismos subyacentes de esta relación no han sido dilucidados (Ammassari et al., 2004; Andrade et al., 2013; Lovejoy y Suhr, 2009). Conocer qué parcelas de la cognición son determinantes en la adherencia, nos permitiría implementar estrategias para contrarrestar los fallos debidos a la disfunción cognitiva (Altice, Kamarulzaman, Soriano, Schechter y Friedland, 2010; Blackstone et al., 2013; Hinkin et al., 2002).

El trastorno por consumo de sustancias tiene una prevalencia muy elevada en pacientes VIH+, en los cuales es especialmente problemático, por varias razones: se asocia directa e indirectamente con un incremento en la transmisión del virus, con un empeoramiento del estado inmunológico independientemente de los factores relacionados con el tratamiento, con un aumento de la morbimortalidad en general, así como con una peor adherencia al TAR (Altice et al., 2010; Parsons, Starks, Millar, Boonrai y Marcotte, 2014). Las sustancias que han demostrado ser factores de riesgo para una mala adherencia son principal, aunque no exclusivamente, alcohol, cannabis, cocaína, anfetaminas y heroína. Sin embargo, los resultados respecto a esta asociación no son homogéneos en todos los estudios (Altice et al., 2010; Waldrop-Valverde et al., 2006). Mientras que existe un consenso bastante extenso sobre la influencia negativa sobre la adherencia al TAR del consumo de alcohol (Azar, Springer, Meyer y Altice, 2010; Gonzalez, Barinas y O'Cleirigh, 2011), y del consumo de cocaína (Meade, Conn, Skalski y Safren, 2011. Rosen et al., 2013); los resultados son dispares para sustancias como el cannabis (González-Álvarez, Madoz-Gúrpide, Parro-Torres, Hernández-Huerta y Ochoa Mangado, 2019; Lovejoy y Suhr, 2009). Respecto a los opioides, el consumo de heroína se ha asociado a una peor adherencia (Azar et al., 2015). Existen resultados contradictorios sobre la influencia en la adherencia de encontrarse en un programa de mantenimiento con metadona (PMM) en usuarios de drogas por vía parenteral (Azar et al., 2015; Cohn et al., 2011). En los pacientes que consumen múltiples sustancias, se ha sugerido que la combinación de alcohol y cannabis podría ser más negativa para la adherencia que el uso exclusivo de alcohol, y a su vez la combinación de alcohol y cocaína podría asociarse con los peores resultados en adherencia (Parsons et al., 2014). El tipo de sustancia y el patrón de consumo podrían ser factores influyentes en esta asociación. Han sido propuestos diversos mecanismos que podrían sustentar esta asociación: un estilo de vida caótico, con horarios inestables (Hinkin et al., 2007; Ingersoll, 2004), un aumento en el olvido de tomas (Anand et al., 2010; Ingersoll, 2004), el fallo en el control de impulsos y la desinhibición favorecidos por el consumo (Anand et al., 2010; Hinkin et al., 2007). Asimismo, algunos pacientes omiten voluntariamente las tomas durante los periodos de consumo, por creerlo incompatible con el tratamiento (Gonzalez et al., 2011; Kalichman et al., 2015).

Por otra parte, el consumo crónico de sustancias puede suponer un incremento en el riesgo de deterioro cognitivo temprano (Altice et al., 2010; Anand et al., 2010; VázquezJusto et al., 2016). Los individuos que consumen opioides muestran déficits en la atención, memoria y función ejecutiva tanto durante su uso activo como en la abstinencia temprana. Además, se ha sugerido que la dependencia a opioides empeora los trastornos neurocognitivos asociados al VIH, porque favorecen la replicación vírica a través de un efecto negativo directo sobre el sistema inmune. Este efecto es aún más evidente en combinación con cocaína (Anand et al., 2010). Existe una mayor disfunción cognitiva y peor adherencia terapéutica en consumidores activos de cocaína respecto a abstinentes. Se ha sugerido que el deterioro cognitivo modera parcialmente esta asociación entre consumo de cocaína y adherencia al TAR (Gonzalez et al., 2011; Meade et al., 2011). Respecto al alcohol, la dependencia puede causar daño cerebral persistente, atrofia subcortical e hipometabolismo en lóbulos frontales. Además, se ha descrito un efecto pernicioso sinérgico del consumo de alcohol y la infección por VIH en el SNC, ya que el deterioro cognitivo asociado al consumo crónico de alcohol es mayor en individuos VIH+ que en VIH- (Anand et al., 2010). Todo ello parece sugerir que existe una interrelación entre el deterioro cognitivo, la adherencia al TAR y el consumo de sustancias (Anand et al., 2010; Hinkin et al., 2007).

El presente trabajo pretende analizar la relación entre el consumo de sustancias y la función cognitiva en la adherencia al TAR en pacientes $\mathrm{VIH}+$. Aunque existe literatura internacional al respecto, las referencias sobre la repercusión del consumo de sustancias y la disfunción cognitiva en la adherencia al TAR son limitadas en el entorno asistencial de la población española (González-Álvarez et al., 2019; Ortego, Huedo-Medina, Vejo y Llorca, 2011). La hipótesis de este estudio es que el consumo de sustancias y el deterioro cognitivo pueden influir negativamente en la adherencia al TAR en pacientes VIH+.

\section{Metodología}

\section{Ámbito y población de estudio}

Un total de 125 pacientes fueron incluidos en este estudio. Previamente al presente artículo, se reportaron datos preliminares de un subconjunto de estos individuos en 
otra publicación de nuestro grupo de trabajo (González-Álvarez et al., 2019). En el citado artículo se estudió la relación entre la adherencia al TAR y el consumo de alcohol, asociado o no al uso de otras sustancias; en este trabajo se introduce, además, el estudio del componente cognitivo.

Constituyeron criterios de inclusión: edad comprendida entre 18 y 65 años, diagnóstico de infección por VIH, encontrarse en seguimiento regular en el Servicio de Enfermedades Infecciosas del Hospital Universitario Ramón y Cajal de Madrid, haber iniciado TAR al menos un año antes de la evaluación, y que este fuera dispensado únicamente a través del Servicio de Farmacia Hospitalaria de dicho hospital. Se consideró seguimiento regular haber acudido al menos a dos citas concertadas en el último año (Tripathi, Youmans, Gibson y Duffus, 2011).

Constituyeron criterios de exclusión: los procesos infecciosos agudos activos, los procesos oncológicos activos, las discrepancias en la clasificación de los pacientes como buenos o malos adherentes entre los diferentes métodos de clasificación utilizados, y el reporte de una tasa de adherencia entre el $90 \%$ y el 95\%. La adherencia al TAR se evaluó mediante el porcentaje de recogida del tratamiento durante el año previo a la evaluación, según el reporte de Farmacia Hospitalaria. Este dato se verificó mediante el cuestionario Simplified Medication Adherence Questionnaire (SMAQ), instrumento de evaluación del cumplimiento terapéutico, validado para su uso en pacientes con VIH (Knobel et al., 2002). En base a ambos parámetros, se clasificaron los pacientes como buenos o malos adherentes, y se informó a su correspondiente médico de referencia del Departamento de Enfermedades Infecciosas del grupo al que había sido asignado cada paciente. Si existía incongruencia entre la adherencia reportada por Farmacia Hospitalaria y el resultado del SMAQ, o si la impresión clínica del profesional de referencia discrepaba del resultado de la clasificación de los dos métodos anteriores, los pacientes eran excluidos. $\mathrm{Al}$ no existir consenso sobre la adherencia mínima efectiva (Viswanathan et al., 2015), se optó por los límites mínimo y máximo más conservadores, con la finalidad de aumentar la potencia de clasificación del método. Por este motivo se excluyeron del estudio aquellos pacientes en los que se reportaron adherencias entre el $90 \%$ y el $95 \%$.

Todos los pacientes incluidos en el estudio fueron informados, aceptaron su participación y firmaron el Consentimiento Informado. El estudio fue aprobado por el Comité Ético de Investigación Clínica del Hospital Universitario Ramón y Cajal.

\section{Tipo y diseño general del estudio}

Se trata de un estudio observacional transversal tipo casos y controles. Los pacientes fueron asignados a cada uno de los grupos en función de la adherencia al TAR. Se consideran "casos" los pacientes con mala adherencia al TAR, definidos, según el reporte de Farmacia Hospitalaria, como aquellos pacientes que hubiesen retirado menos del $90 \%$ de las tomas del TAR durante el último año. Conforman los "controles" pacientes con la misma procedencia, que, en el momento de entrar al estudio, se diferenciaban exclusivamente por su buena adherencia al TAR, definida como aquellos que hubiesen retirado más del $95 \%$ de las tomas durante el último año (Paterson et al., 2000).

\section{Procedimiento de recogida de la muestra:}

La selección de los casos se realizó a través de un muestreo consecutivo de un listado de pacientes no adherentes generado de forma ordinaria y con frecuencia mensual por Farmacia Hospitalaria. Los controles fueron seleccionados por muestreo consecutivo del listado de pacientes citados en la consulta monográfica de VIH del Servicio de Enfermedades Infecciosas. La recogida de muestra tuvo lugar entre mayo 2013 y septiembre 2015.

\section{Procedimiento de recogida de datos:}

La recogida de variables se implementó según un protocolo de historia clínica semi-estructurada especialmente diseñada para esta investigación, en el que se incluyó la escala SMAQ. Asimismo, fue incluida en el protocolo una batería de pruebas neuropsicológicas validadas para la población española. entre las que se encontraban el Trail Making Test (TMT) y el Test del Mapa del Zoo.

- El Test del Mapa del Zoo forma parte de la batería de exploración neuropsicológica Behavioral Assesment of the Dysexecutive Syndrome (BADS). Esta herramienta es usada especialmente para medir la habilidad de planificación como parte de las funciones ejecutivas. La planificación puede definirse como la habilidad para organizar la conducta en relación a un objetivo específico que debe ser alcanzado a través de una serie de pasos intermedios. Se ha sugerido que es un proceso que consta de dos pasos: formulación y ejecución. La formulación es la habilidad mental para desarrollar una estrategia lógica que guíe el curso de acción, mientras que la ejecución es la competencia de llevar a cabo el plan desarrollado en la formulación (Oosterman, Wijers y Kessels, 2013).

- El TMT es uno de los instrumentos de mayor difusión y uso en las evaluaciones neuropsicológicas que pretenden medir velocidad de procesamiento y funcionalidad ejecutiva. Diversos estudios coinciden en afirmar que tiene una estructura compleja que engloba diversos mecanismos cognitivos. La búsqueda visual, la velocidad perceptiva/motora, la velocidad de procesamiento, la memoria de trabajo y la inteligencia general son algunos de los constructos más frecuentemente citados que se piensa contribuyen a la realización de la prueba. Consta de dos partes: la A examina principalmente habilidades visoperceptivas y la $\mathrm{B}$ implica, principalmente, la memoria de trabajo 
y secundariamente, la flexibilidad para el cambio de tarea (Sánchez-Cubillo et al., 2009).

\section{Definición de las variables del estudio}

Se recogieron variables sociodemográficas, como edad, sexo, raza, estado civil, nivel socioeconómico, nivel educativo y laboral. Con el objetivo de aumentar la potencia estadística del análisis, algunas variables fueron recodificadas, preservando un sentido clínico. Se consideró profesión no cualificada las categorías de ausencia de profesión, ama de casa, estudiante y obrero no cualificado; mientras que se clasificaron como cualificados los obreros con cualificación laboral, empleados, funcionarios y profesiones liberales. Respecto al nivel sociodemográfico, se consideró riesgo económico el hecho de acumular deudas, no poder afrontar pagos o necesidad de ayuda externa a su unidad familiar.

Asimismo, se incluyeron variables de la infección por VIH y su tratamiento: coinfección por Virus de la Hepatitis C (VHC), tiempo en seguimiento, tiempo en tratamiento con TAR, número de tomas de medicación al día, número de pastillas al día y presencia de figura de supervisión del tratamiento, entre otras. Se describió la historia de consumo de sustancias en los 12 meses previos a la evaluación (alcohol, cocaína, heroína, cannabis, estimulantes y benzodiacepinas, además del tratamiento con metadona). Se categorizó el consumo de alcohol en consumo perjudicial y consumo no problemático, según los criterios de la Décima Revisión de la Clasificación Internacional de Enfermedades (Organización Mundial de la Salud [O.M.S.], 1992). Para el resto de sustancias, se incluyó como positivo cualquier patrón de consumo, si bien todos los individuos que admitieron consumo presentaban frecuencia al menos semanal. Se recogió también la presencia de conductas de riesgo asociadas al uso de sustancias por vía parenteral: compartir jeringuillas y reutilizarlas. Respecto a las pruebas neuropsicológicas, en el Test del Mapa del Zoo se recogió la variable principal "puntuación total” y cuatro variables secundarias: "número de aciertos", "número de errores", "tiempo de planificación" y "tiempo total de realización de la prueba”. Se definieron las variables "puntuación TMT-A" y "puntuación TMT-B", en ambos casos la puntuación se obtuvo directamente según el tiempo empleado en completar la tarea.

\section{Análisis estadístico}

Se realizó en primer lugar un análisis bruto de los resultados. Se comprobó que las variables cuantitativas seguían una distribución normal mediante el test de KolmogórovSmirnov y la visualización de las gráficas. En los casos de discrepancia, se siguió la información gráfica. Respecto al análisis descriptivo, en las variables que seguían una distribución normal, los resultados se expresaron como media y desviación típica. En las variables no paramétricas, se utilizaron la mediana y el rango intercuartílico entre $25 \%$ y $75 \%$. En las variables cualitativas, el estadístico descriptivo fue el porcentaje. La comparación entre casos y controles se realizó a través del estadístico Chi-cuadrado de Pearson en las variables cualitativas. Para las variables cuantitativas que seguían una distribución normal se recurrió al estadístico $t$ de Student para muestras independientes, y para aquellas en las que al menos uno de los grupos no seguía una distribución normal, se utilizó el estadístico U de Mann Whitney.

Por último, se realizó un análisis multivariante mediante regresión logística binaria por el método de "pasos hacia atrás" (razón de verosimilitud). Inicialmente fueron incluidas variables que habían demostrado estar relacionadas con la adherencia al TAR en el análisis bruto, y que, a su vez, cumplían el criterio de plausibilidad biológica, y tenían relación con el objeto de nuestro estudio. También se incluyeron variables que habían demostrado ser interesantes en la literatura previa. Las variables introducidas en el análisis multivariante fueron las siguientes: edad, género, nivel socioeconómico, nivel educativo, número de pastillas prescritas al día, conductas de riesgo en relación a compartir jeringuillas, presencia de supervisión de la mediación por parte de terceros, coinfección con VHC, consumo de sustancias (alcohol, cannabis, cocaína, heroína y tratamiento con metadona) y puntuación en pruebas psicométricas (Zoo y TMT-B).

Se consideró la significación de los datos a partir de un valor de $\mathrm{p}<0,05$.

\section{Resultados}

\section{Resultados del análisis descriptivo y del análisis bruto}

Descripción general de la muestra y análisis comparativo de variables sociodemográficas y clínicas. Se analizó una muestra total de 125 sujetos, 79 controles y 46 casos. De ellos, el $68 \%$ fueron varones y el $84 \%$ de raza caucásica. La mediana de edad de la muestra fue 48,4 años (RIQ: 43,6 - 52,8).

No se demostró diferencia estadísticamente significativa entre casos y controles en cuanto a las variables sexo, edad, raza y estado civil. La ausencia de diferencia significativa entre el grupo control y el grupo de casos en la variable edad la excluye como un posible factor de confusión en relación al deterioro cognitivo. Sí se halló diferencia significativa en el nivel académico, ya que la frecuencia de estudios superiores fue mayor en los controles $(p=0,001)$. Asimismo, los casos tenían una menor cualificación profesional ( $p=0,027)$, y un peor nivel socioeconómico $(\mathrm{p}<$ 0,001). Tanto el nivel académico como el nivel socioeconómico fueron incluidos en el análisis multivariante con el objetivo de estudiar la hipótesis de nuestro estudio con independencia de la posible influencia de estas variables no homogéneas entre casos y controles. La cualificación laboral no fue incluida por su menor significación estadística y por la necesidad de limitar el número de variables introducidas en el análisis multivariante de forma proporcionada a nuestro tamaño muestral. No se encontraron diferencias 
Tabla 1. Análisis comparativo de variables sociodemográficas.

\begin{tabular}{|c|c|c|c|c|}
\hline & & $\begin{array}{c}\text { Controles } \\
n(\%)\end{array}$ & $\begin{array}{l}\text { Casos } \\
\mathrm{n}(\%)\end{array}$ & $\begin{array}{c}\mathbf{X}^{2} \\
p\end{array}$ \\
\hline \multirow{2}{*}{ Sexo } & Varón & $52(65,8 \%)$ & $33(71,7 \%)$ & \multirow{2}{*}{0,494} \\
\hline & Mujer & $27(34,2 \%)$ & $13(28,3 \%)$ & \\
\hline \multirow{2}{*}{ Raza } & Caucásica & $70(88,6 \%)$ & $35(76,1 \%)$ & \multirow{2}{*}{0,066} \\
\hline & Otras & $9(11,4 \%)$ & $11(23,9 \%)$ & \\
\hline \multirow{2}{*}{ Estado civil } & En pareja & $28(35,4 \%)$ & $18(39,1 \%)$ & \multirow{2}{*}{0,680} \\
\hline & Otros & $51(64,6 \%)$ & $28(60,9 \%)$ & \\
\hline \multirow{2}{*}{ Nivel académico } & Primaria/Graduado & $32(40,5 \%)$ & $33(71,7 \%)$ & \multirow{2}{*}{0,001} \\
\hline & Bachiller/FP*/Universidad & $47(59,5 \%)$ & $13(28,3 \%)$ & \\
\hline \multirow{2}{*}{ Profesión } & No cualificada & $31(40,3 \%)$ & $28(60,9 \%)$ & \multirow{2}{*}{0,027} \\
\hline & Cualificada & $46(59,7 \%)$ & $18(39,1 \%)$ & \\
\hline \multirow{2}{*}{ Nivel socioeconómico } & Sin problemas económicos & $69(87,3 \%)$ & $21(45,7 \%)$ & \multirow{2}{*}{$<0,001$} \\
\hline & Riesgo económico & $10(12,7 \%)$ & $25(54,3 \%)$ & \\
\hline \multirow{2}{*}{$\begin{array}{l}\text { Supervisión del } \\
\text { tratamiento }\end{array}$} & Nunca & $71(89,9 \%)$ & $30(65,2 \%)$ & \multirow{2}{*}{0,001} \\
\hline & A veces/siempre & $8(10,1 \%)$ & $16(34,8 \%)$ & \\
\hline
\end{tabular}

Nota. *FP: Formación profesional.

Tabla 2. Análisis comparativo de variables clínicas.

\begin{tabular}{lccc}
\hline & $\begin{array}{c}\text { Controles } \\
\text { Mediana (RIQ*) }\end{array}$ & $\begin{array}{c}\text { Casos } \\
\text { Mediana (RIQ*) }\end{array}$ & $\begin{array}{c}\text { U de Mann } \\
\text {-Whitney } \\
p\end{array}$ \\
\hline Edad (años) & $49,1(45,3-53,4)$ & $46,4(6,3)^{\star}$ & 0,079 \\
$\begin{array}{l}\text { Tiempo en } \\
\text { seguimiento } \\
\text { (años) }\end{array}$ & $15,0(8,0-21,0)$ & $18,0(9,0-22,0)$ & 0,125 \\
$\begin{array}{l}\text { Tiempo en } \\
\text { tratamiento } \\
\text { (años) }\end{array}$ & $13,0(5,0-19,0)$ & $16,5(8,0-20,0)$ & 0,176 \\
$\begin{array}{l}\text { Número de } \\
\text { pastillas/día }\end{array}$ & $2,0(1,0-3,0)$ & $3,0(2,7-4,0)$ & $\mathbf{0 , 0 0 2}$ \\
$\begin{array}{l}\text { Número de } \\
\text { tomas/día }\end{array}$ & $1,0(1,0-2,0)$ & $1,0(1,0-2,0)$ & 0,866 \\
\hline
\end{tabular}

Nota. ${ }^{*}$ Rango intercuartílico (Q25 - Q75); * Media (Desviación estándar).

entre casos y controles para las variables tiempo en tratamiento, tiempo en seguimiento y número de tomas de medicación al día. Sí existió una diferencia estadísticamente significativa en el número de pastillas que componían el tratamiento diario entre ambos grupos, que es mayor en los casos $(\mathrm{p}=0,002)$. También resultó significativa la mayor necesidad en los casos de supervisión del TAR por parte de terceros $(p=0,001)$ (Tablas 1 y 2$)$.

La coinfección por el VHC fue significativamente mayor en el grupo de los casos $(70,5 \%$ frente al $34,6 \%$ de los controles, $\mathrm{p}<0,001)$. Asimismo, los casos presentaron mayores conductas de riesgo en relación a compartir jeringuillas $(58,7 \%$ frente a $26,6 \%, \mathrm{p}<0,001)$.
Tabla 3. Análisis comparativo de variables relacionadas con el consumo de sustancias en el último año.

\begin{tabular}{lccc}
\hline & $\begin{array}{c}\text { Controles } \\
\mathrm{n}(\%)\end{array}$ & $\begin{array}{c}\text { Casos } \\
\mathrm{n}(\%)\end{array}$ & $\begin{array}{c}\mathbf{X}^{2} \\
p\end{array}$ \\
\hline Cualquier sustancia ilegal & $24(30,4 \%)$ & $31(67,4 \%)$ & $<\mathbf{0 , 0 0 1}$ \\
Alcohol (problemático) & $12(15,2 \%)$ & $22(47,8 \%)$ & $<\mathbf{0 , 0 0 1}$ \\
Cannabis & $24(30,4 \%)$ & $19(41,3 \%)$ & 0,215 \\
Cocaína & $14(17,7 \%)$ & $21(45,7 \%)$ & $\mathbf{0 , 0 0 1}$ \\
Otros estimulantes & $0(0 \%)$ & $3(6,5 \%)$ & $\mathbf{0 , 0 2 2}$ \\
Heroína & $3(3,8 \%)$ & $7(15,2 \%)$ & $\mathbf{0 , 0 2 3}$ \\
Tratamiento con metadona & $5(6,3 \%)$ & $16(34,8 \%)$ & $<\mathbf{0 , 0 0 1}$ \\
Benzodiazepinas & $2(2,5 \%)$ & $10(21,7 \%)$ & $<\mathbf{0 , 0 0 1}$ \\
\hline
\end{tabular}

Análisis comparativo de variables relacionadas con el consumo de sustancias en el último año. El 67,4\% de los casos y el 30,4\% de los controles presentaron consumo de sustancias ilegales en el último año, siendo esta diferencia estadísticamente significativa $(\mathrm{p}<0,001)$. El consumo problemático de alcohol fue mayor en los casos $(p<0,001)$. Igualmente fue más frecuente entre los casos el consumo de cocaína ( $\mathrm{p}=$ $0,001)$, de otros estimulantes $(\mathrm{p}=0,022)$ y de heroína $(\mathrm{p}=$ 0,023). El número de sujetos que se encontraban en PMM para la adicción a opiáceos fue significativamente mayor en los casos $(p<0,001)$. También el abuso de benzodiacepinas fue estadísticamente más frecuente entre los casos ( $p$ $<0,001)$. No se hallaron diferencias significativas respecto al consumo de cannabis (Tabla 3). 
Análisis comparativo de variables relacionadas con las pruebas neuropsicológicas. En el Test del Mapa del Zoo se encontraron diferencias significativas entre casos y controles en cuatro de las cinco variables que contemplamos en nuestra corrección, siempre con mejor rendimiento en los controles: en puntuación total $(\mathrm{p}=0,002)$, en número de aciertos $(p=0,045)$, en número de errores $(p=0,014)$, y en tiempo total de la realización de la prueba $(p=0,038)$. Respecto al TMT, en ambas partes los casos tardan significativamente más tiempo en completar las pruebas que los controles (TMT-A: $\mathrm{p}=0,003$ y TMT-B: $\mathrm{p}=0,001$ ) (Tabla 4).

\section{Análisis ajustado de variables relacionadas con la adherencia al TAR. Regresión logística}

De los 125 sujetos de la muestra total, se incluyeron en el análisis ajustado 111 (88,8\%). De ellos, 74 fueron controles y 37 fueron casos. La pérdida de 14 sujetos se debe a que estos tenían incompleta alguna de las variables que fueron introducidas en el análisis, en concreto la puntuación en

Tabla 4. Análisis comparativo de las variables relacionadas con las pruebas neuropsicológicas.

\begin{tabular}{|c|c|c|c|c|}
\hline & & $\begin{array}{c}\text { Controles } \\
\text { Mediana (RIQ*) }\end{array}$ & $\begin{array}{c}\text { Casos } \\
\text { Mediana }\left(R^{*} Q^{*}\right)\end{array}$ & $\begin{array}{c}\text { U de Mann } \\
\text { Witney } \\
p\end{array}$ \\
\hline \multirow{2}{*}{$\begin{array}{l}\text { Trial } \\
\text { Making } \\
\text { Test }\end{array}$} & $\begin{array}{l}\text { TMT-A } \\
\text { (segundos) }\end{array}$ & $\begin{array}{c}41,0 \\
(34,0-54,0)\end{array}$ & $\begin{array}{c}53,5 \\
(39,5-74,2)\end{array}$ & 0,003 \\
\hline & $\begin{array}{l}\text { TMT-B } \\
\text { (segundos) }\end{array}$ & $\begin{array}{c}85,0 \\
(67,0-124,5)\end{array}$ & $\begin{array}{c}143,0 \\
(86,0-200,0)\end{array}$ & 0,001 \\
\hline \multirow{5}{*}{$\begin{array}{l}\text { Test del } \\
\text { Mapa } \\
\text { del Zoo }\end{array}$} & № aciertos & $\begin{array}{c}8,0 \\
(7,0-8,0)\end{array}$ & $\begin{array}{c}7,0 \\
(7,0-8,0)\end{array}$ & 0,045 \\
\hline & № errores & $\begin{array}{c}1,0 \\
(0,0-1,0)\end{array}$ & $\begin{array}{c}1,0 \\
(0,0-3,0)\end{array}$ & 0,014 \\
\hline & $\begin{array}{l}\text { Puntuación } \\
\text { total }\end{array}$ & $\begin{array}{c}7,0 \\
(6,0-8,0)\end{array}$ & $\begin{array}{c}5,0 \\
(3,5-5,0)\end{array}$ & 0,002 \\
\hline & $\begin{array}{l}\text { Tiempo de } \\
\text { planificación } \\
\text { (segundos) }\end{array}$ & $\begin{array}{c}75,0 \\
(39,0-146,0)\end{array}$ & $\begin{array}{c}70,0 \\
(22,5-121,0)\end{array}$ & 0,205 \\
\hline & $\begin{array}{l}\text { Tiempo total } \\
\text { (segundos) }\end{array}$ & $\begin{array}{c}145,0 \\
(89,0-203,5)\end{array}$ & $\begin{array}{c}187,5 \\
(126,0-240,0)\end{array}$ & 0,038 \\
\hline
\end{tabular}

Nota. ${ }^{*}$ Rango intercuartílico $\left(Q_{25}-\mathrm{Q} 75\right)$.
TMT-B y/o la puntuación total en el Zoo Map. El motivo de pérdida es el abandono de la entrevista con anterioridad a su finalización, en relación posiblemente a la fatiga del paciente y a una duración prolongada de aquella. Se realizaron modelos de imputación múltiple de los valores perdidos, que no mostraron cambios de interés ni mejoría significativa del modelo final; por este motivo se decidió asumir las pérdidas.

El modelo final clasifica correctamente al $82,9 \%$ de la muestra global, con mayor especificidad que sensibilidad (prueba de Hosmer y Lemeshow: 90,5\% de los controles y $67,6 \%$ de los casos); y explica un $53,4 \%$ de la variable adherencia.

En la Tabla 5 se indican las variables que muestran relación con la adherencia al TAR de forma estadísticamente significativa.

Datos sociodemográficos y clínicos. Según los resultados obtenidos, aumentan el riesgo de mala adherencia: una menor edad, el género masculino, un nivel socioeconómico bajo, una pauta de TAR consistente en un mayor número de pastillas al día y la coinfección por VHC. En nuestro modelo, no han demostrado asociación con mala adherencia la conducta de riesgo consistente en compartir jeringuillas, la necesidad de supervisión del tratamiento ni el nivel educativo del paciente.

Consumo de sustancias. El consumo perjudicial de alcohol incrementa por 3,398 (IC95\% 1,040 - 11,100) el riesgo de ser clasificado como mal adherente, frente a la situación de abstinencia o consumo no problemático, una vez ajustado por las variables señaladas en la Tabla 5. Sin embargo, los consumos de cannabis, cocaína o heroína y el uso terapéutico de metadona no se asocian significativamente con un empeoramiento en la adherencia terapéutica.

Pruebas neuropsicológicas. La puntuación total obtenida en el Mapa del Zoo, tras el ajuste por las variables señaladas en la Tabla 5, se relaciona significativamente con la adherencia al TAR. Cada punto adicional en la puntuación de dicho

Tabla 5. Regresión logística, método "Introducir".

\begin{tabular}{|c|c|c|c|c|c|c|c|}
\hline & B & Error estándar & Wald & $\begin{array}{l}\text { Grados } \\
\text { libertad }\end{array}$ & $p$ & $\operatorname{Exp}(B)$ & IC $95 \%$ para $\operatorname{Exp}(B)$ \\
\hline Edad & $-0,110$ & 0,046 & 5,852 & 1 & 0,016 & 0,896 & $0,819-0,979$ \\
\hline Sexo & $-1,338$ & 0,655 & 4,173 & 1 & 0,041 & 0,262 & $0,073-0,947$ \\
\hline Número de pastillas & 0,514 & 0,211 & 5,938 & 1 & 0,015 & 1,672 & $1,106-2,528$ \\
\hline Nivel socioeconómico & 1,296 & 0,637 & 4,136 & 1 & 0,042 & 3,654 & $1,048-12,737$ \\
\hline Coinfección VHC & 1,821 & 0,662 & 7,563 & 1 & 0,006 & 6,178 & $1,687-22,620$ \\
\hline Alcohol & 1,223 & 0,604 & 4,103 & 1 & 0,043 & 3,398 & $1,040-11,100$ \\
\hline Zoo Puntuación total & $-0,417$ & 0,142 & 8,662 & 1 & 0,003 & 0,659 & $0,500-0,870$ \\
\hline Constante & $-0,463$ & 2,497 & 0,034 & 1 & 0,853 & 0,630 & \\
\hline
\end{tabular}


test multiplica por 0,659 la probabilidad de ser considerado mal adherente. A la inversa, cada punto adicional multiplica por 1,517 (IC95\% 1,149 - 2,000) la probabilidad de ser considerado buen adherente. Sin embargo, no se ha encontrado relación estadísticamente significativa entre la puntuación obtenida en el TMT-B y la adherencia.

\section{Discusión}

El objetivo del presente estudio es estudiar si el consumo de sustancias y el deterioro cognitivo, evaluado mediante el Test del Mapa del Zoo y el TMT; influyen negativamente en la adherencia al TAR en pacientes VIH+. Los resultados obtenidos confirman parcialmente nuestra hipótesis de partida.

Las diferencias en la ejecución de las pruebas neuropsicológicas entre casos y controles fueron significativas para casi la totalidad de las variables estudiadas. En el análisis bruto del Test del Mapa del Zoo se halló diferencia en cuatro de las cinco variables contempladas. No se observó diferencia significativa en la variable tiempo de planificación entre casos y controles, aunque el tiempo total de ejecución sí es significativamente mayor en los malos adherentes. Esto puede deberse a que los casos, en promedio, comenzaban la tarea de forma impulsiva y sobreestimando su capacidad de resolución, y sin embargo luego requerían más tiempo para completar la prueba y cometían más errores. De las variables neuropsicológicas introducidas en el análisis multivariante (TMT-B y puntuación total en el Mapa del Zoo) esta última fue la única que mantuvo la asociación estadísticamente significativa con la variable adherencia en el modelo final de regresión logística, tras el ajuste por el resto de variables. El TAR suele ser una pauta compleja cuyo adecuado cumplimiento precisa de habilidades de planificación y ejecución; por tanto, parece coherente que una dificultad en la planificación, medida por el Test del Mapa del Zoo, repercuta en una peor capacidad de adherirse a dicha pauta. Estos resultados son congruentes con los hallados otros estudios, en los que se ha demostrado asociación significativa entre una alteración en las funciones ejecutivas y una peor adherencia al TAR (Andrade et al., 2013; Waldrop-Valverde et al., 2006).

Resulta también significativo que los casos obtuvieron peores rendimientos que los controles en la ejecución de ambas versiones del TMT en el análisis bruto. Esto respalda los resultados obtenidos en estudios previos que relacionan un déficit en la memoria de trabajo, analizada mediante el TMT, con una peor adherencia al TAR (Wagner, 2002). No obstante, existe mayor respaldo en la literatura para la asociación entre la adherencia y la versión B del TMT (Solomon y Halkitis, 2008; Thaler et al., 2015). Debido a la necesidad de introducir un número de variables proporcionado para nuestro tamaño muestral, se decidió no incluir la versión A del TMT en el análisis multivarian- te. Aun así, el TMT-B no demostró significación estadística en el modelo final, posiblemente porque existe un solapamiento entre las áreas evaluadas por el Mapa del Zoo y el TMT-B.

La asociación entre consumo de alcohol y peor adherencia se mantiene en nuestro modelo de regresión logística tras el ajuste por otros consumos, por lo que el alcohol sería un factor de riesgo para una mala adherencia independientemente del uso de otras sustancias. Según los resultados que arroja el análisis bruto, el consumo de heroína, cocaína y otros estimulantes son significativamente mayores en los malos adherentes; sin embargo, la asociación no se mantiene en el modelo multivariante. Este último hallazgo contrasta con los resultados obtenidos en otros estudios: Azar et al. (2015) encontraron una relación significativa entre una peor adherencia y el uso de cocaína y heroína intravenosas, independientemente; y Rosen et al. (2013) sugieren un efecto negativo del consumo de alcohol, heroína, cocaína y otros estimulantes sobre la adherencia. Es posible que el no hallazgo de dicha relación en nuestro estudio se deba al escaso número de sujetos que reportan dichos consumos, que los pacientes con consumos más graves no estén representados en nuestra muestra, o que exista un solapamiento entre consumo de alcohol y estas sustancias. No se ha encontrado diferencia significativa en el consumo de cannabis entre casos y controles, lo que respalda los resultados de estudios previos (Rosen et al., 2013; Slawson et al., 2015). Respecto a los programas de PMM en usuarios de drogas por vía parenteral, son estadísticamente más frecuentes en malos adherentes en el análisis bruto, pero no resulta significativa esta asociación en el análisis multivariante. Este hallazgo difiere de los sugerido por otros autores (Malta, Strathdee, Magnanini y Bastos, 2008), y podría deberse a que, en nuestra muestra, el perfil de paciente en PMM se corresponde con un peor nivel socioeconómico y situación clínica, lo cual sería congruente con los hallazgos de otros estudios (Martínez-Luna et al., 2018; Pedrero-Pérez y Grupo MethaQoL, 2017).

Los resultados muestran que la coinfección por VHC fue significativamente más frecuente entre los pacientes con mala adherencia. Esta variable no ha demostrado influir en la adherencia al TAR en otros estudios anteriores (Shuper et al., 2016). Es posible que la coinfección por VHC se asocie a factores que dificulten la adherencia: mayor presencia de hepatotoxicidad, menor eficacia del TAR, mayor prevalencia del consumo de sustancias, peor rendimiento cognitivo y mayor marginalización social. No obstante, esta posible relación excede el objetivo de nuestro trabajo, siendo precisos otros estudios más específicos para caracterizarla. Los pacientes con peor nivel socioeconómico presentan mayor probabilidad de ser malos adherentes en el análisis multivariante, hallazgo que coincide con estudios anteriores (Tsuyuki y Surratt, 2015). Como ya se 
había sugerido por otros autores (Nachega et al., 2014), en nuestro estudio las pautas de TAR consistentes en mayor número de pastillas, se relacionan con una peor adherencia. No obstante, resulta plausible que esta situación se retroalimente, de manera que un peor estado inmunológico, relacionado con una peor adherencia y con las resistencias desarrolladas en ese contexto, haga necesaria una pauta de tratamiento más compleja, que a su vez dificulta más la adherencia. Por otra parte, no existe diferencia entre el número de tomas al día, lo cual probablemente refleja un esfuerzo de los profesionales en simplificar las pautas de tratamiento, a pesar de que los casos precisan mayor número de pastillas al día.

De los datos obtenidos en nuestro trabajo, se deduce que el consumo perjudicial de alcohol y un peor funcionamiento cognitivo ejecutivo, medido por el Mapa del Zoo, se relacionan significativamente con una mala adherencia al TAR. Este hallazgo corrobora en el entorno de la población española gran parte de los resultados publicados en la literatura internacional (Andrade et al., 2013; Azar et al., 2010; Thaler et al., 2015). Respecto a nuestro país, en el metaanálisis realizado por Ortego et al. (2011) se encontró relación entre la mala adherencia y el consumo de alcohol y de otras sustancias, pero únicamente en el modelo univariante; el deterioro cognitivo no fue estudiado. En el estudio previo realizado por nuestro grupo (González-Álvarez et al., 2019) se halló que el consumo perjudicial de alcohol constituye un factor de riesgo de mala adherencia al TAR, de manera independiente al consumo de otras sustancias.

Parte de las limitaciones de este estudio pueden estar relacionadas con la procedencia de la muestra: una consulta externa del Servicio de Enfermedades Infecciosas de un hospital que constituye un centro de referencia para el tratamiento del VIH. Es posible que, por el contexto asistencial, se trate de una población envejecida, con elevado tiempo promedio de seguimiento y tratamiento, y en la que estarían excluidos los casos más graves, que tendrían mayor dificultad para mantener un seguimiento regular en la consulta. Por otra parte, la pérdida de sujetos en el análisis multivariante, aunque pequeña, puede haber supuesto un sesgo de exclusión de los perfiles más deteriorados, que pueden haber presentado más dificultades para completar los test.

Los instrumentos empleados en este trabajo para estudiar el deterioro cognitivo miden particularmente las funciones ejecutivas. En este sentido, otra posible limitación podría ser no haber ampliado la exploración a otros dominios cognitivos.

Respecto al consumo de sustancias, no se utilizaron métodos para corroborar los criterios de abuso/dependencia o trastorno por consumo, solamente se registró, mediante entrevista con el paciente, la presencia o ausencia de consumo en el último año, y no se realizaron pruebas de cribado en muestras biológicas. Por otra parte, la prevalencia del consumo de heroína, cocaína y otros estimulantes es escasa en la muestra, lo cual puede haber limitado la capacidad de evaluación del efecto real de dichas variables en la adherencia. Asimismo, es posible que exista un sesgo respecto a los pacientes con patrones de consumo más graves, los cuales pueden no haber sido incluidos por no cumplir el criterio de un seguimiento ambulatorio regular. Por todo ello, los resultados son únicamente extrapolables a una población de perfil similar. Sería necesario estudiar la repercusión del deterioro cognitivo y el consumo de sustancias en la adherencia al TAR en poblaciones caracterizadas por consumos más graves, mayor deterioro psicosocial y menor acceso a recursos sanitarios, para conocer la naturaleza de esta asociación en pacientes de ese perfil.

Otra posible limitación es que el método de cuantificación de la adherencia por parte del Servicio de Farmacia Hospitalaria se basa en la retirada de la medicación, no estrictamente en su consumo. Por tanto, estarían sesgados en la recogida de información los defectos en el horario de las tomas, la duplicidad de tomas o el extravío de medicación. No obstante, una posible fortaleza del estudio es que este recuento se comprueba mediante dos métodos complementarios: el instrumento SMAQ y la opinión del profesional responsable. Este diseño podría disminuir el riesgo de sobreestimar la adherencia y aumentar la probabilidad de una correcta clasificación de los pacientes (Henegar et al., 2015).

La importancia de una adecuada adherencia al TAR ya ha sido ampliamente descrita como medio para reducir la morbimortalidad asociada al VIH (Lovejoy y Suhr, 2009; Thaler et al., 2015). Los pacientes con disfunción cognitiva, así como aquellos que consumen sustancias ilegales y/o alcohol de forma problemática, tienen más riesgo de ser malos adherentes. Como ya se había sugerido en la literatura previa, estas variables actúan como factores de riesgo independientes, cuyos efectos combinados son sumatorios para una mala adherencia (Moore et al., 2012; Thaler et al., 2015). No obstante, el presente estudio no permite dilucidar los mecanismos que sustentan esta asociación. Posiblemente el deterioro cognitivo sea, en parte, un mediador entre el consumo de sustancias y la adherencia al TAR, como se ha sugerido en estudios previos (Gonzalez et al., 2011; Meade et al., 2011). Sería preciso realizar estudios con mayor tamaño muestral y diferente diseño para plantear hipótesis más específicas al respecto.

Es fundamental, por una parte, interrogar sobre la existencia y patrón del consumo de sustancias, con el objetivo de establecer medidas que disminuyan la influencia del consumo y facilitar el acceso a recursos terapéuticos (Gonzalez et al., 2011; Parsons et al., 2014). Por otra parte, es necesaria la detección del deterioro cognitivo en la práctica clínica habitual, pero actualmente no existe una batería neuropsicológica específica para pacientes VIH+ 
(GeSIDA y SPNS, 2014; Muñoz-Moreno et al., 2014), en este sentido, el TMT-B y el Test del Mapa del Zoo pueden ser herramientas de cribado útiles y de fácil aplicación (GeSIDA y SPNS, 2014; Oosterman et al., 2013; Sánchez-Cubillo et al., 2009). Estudios anteriores han demostrado que las pautas de tratamiento más complejas se relacionan con una peor adherencia, particularmente en individuos con déficits cognitivos (Hinkin et al., 2002). En los pacientes en los que resulte positivo un cribado de deterioro de las funciones cognitivas, es posible que la simplificación del tratamiento favoreciera un mejor cumplimiento terapéutico. Asimismo, las estrategias de estimulación cognitiva, el trabajo motivacional y el uso de dispositivos de apoyo ambientales (alarmas, listas, implicación de parientes o terceras personas...), entre otros, se han sugerido como estrategias potencialmente beneficiosas en estos pacientes (Bragança y Palha, 2011; Parsons et al., 2014; Tran et al., 2013).

En conclusión, el estudio muestra la importancia de la disfunción cognitiva y el consumo alcohol, una vez ajustados por el resto de variables, como factores de riesgo independientes de una mala adherencia al TAR. Por otra parte, variables sociodemográficas y clínicas, como el sexo masculino, la juventud, un bajo nivel socioeconómico, la coinfección con VHC o un número elevado de pastillas, se asocian también a una peor adherencia. La relevancia de estos resultados en la práctica clínica radica en la importancia de ampliar el conocimiento sobre los factores que modulan la adherencia al TAR, con los objetivos de implementar estrategias de prevención primaria y secundaria.

Es necesaria la intervención sobre los factores de riesgo modificables con el objetivo de minimizar el incumplimiento terapéutico en pacientes VIH. El cribado y la detección del consumo de sustancias podría ayudar a implementar medidas para facilitar el acceso a dispositivos específicos de tratamiento. Asimismo, la detección sistemática de déficits en el funcionamiento cognitivo permitiría establecer estrategias para mejorar el cumplimiento terapéutico. La mejora de la adherencia terapéutica, a su vez, constituye una medida preventiva para el desarrollo de deterioro cognitivo y la disminución de la morbimortalidad asociada al VIH.

\section{Conflicto de intereses}

Los autores declaran que no existe ningún conflicto de interés para el presente trabajo. Carlos Parro Torres declara que en los últimos años ha recibido financiación como ponente y ha colaborado en proyectos de Lundbeck, Servier y Janssen. Daniel Hernández Huerta declara que en los últimos años ha recibido financiación como ponente y ha colaborado en proyectos de Otsuka y Janssen. Enriqueta Ochoa Mangado declara que en los últimos años ha recibido financiación como ponente y ha colaborado en proyectos de Lundbeck, Servier, Reckitt Benckiser/Indivior y Ferrer-Brainfarma.

\section{Referencias}

Altice, F.L., Kamarulzaman, A., Soriano, V.V., Schechter, M. y Friedland G.H. (2010). Treatment of medical, psychiatric, and substance-use comorbidities in people infected with HIV who use drugs. The Lancet, 376, 367-387. doi:10.1016/S0140-6736(10)60829-X.

Ammassari, A., Trotta, M.P., Murri, R., Castelli, F., Narciso, P, Noto, P., ... Antinori, A. (2002). Correlates and predictors of adherence to highly active antiretroviral therapy: overview of published literature. Journal of Acquired Immune Deficiency Syndromes, 31, S123-S127.

Ammassari, A., Antinori, A., Aloisi, M.S., Trotta, M.P., Murri, R., Bartoli, L., ... Starace, F. (2004). Depressive symptoms, neurocognitive impairment, and adherence to highly active antiretroviral therapy among HIV-infected patients. Psychosomatics, 45, 394-402. doi:10.1176/ appi.psy.45.5.394.1.

Anand, P., Springer, S.A., Copenhaver, M.M. y Altice, F.L. (2010). Neurocognitive impairment and HIV risk factors: a reciprocal relationship. AIDS and Behavior, 14, 1213-1226. doi:10.1007/s10461-010-9684-1.

Andrade, A.S.A., Deutsch, R., Celano, S., Duarte N.A., Marcotte, T.D., Umlauf, A., ... Collier, A.C. (2013). Relationships among neurocognitive status, medication adherence measured by pharmacy refill records, and virologic suppression in HIV-infected persons. Journal of Acquired Immune Deficiency Syndromes, 62, 282-292. doi:10.1097/QAI.0b013e31827ed678.

Antinori, A., Arendt, G., Becker, J.T., Brew, B.J., Byrd, D.A., Cherner, M., ... Wogna, V.E. (2007). Updated research nosology for HIV-associated neurocognitive disorders. Neurology, 69, 1789-1799. doi:10.1212/01. WNL.0000287431.88658.8b.

Azar, M.M., Springer, S.A., Meyer, J.P. y Altice, F.L. (2010). A systematic review of the impact of alcohol use disorders on HIV treatment outcomes, adherence to antiretroviral therapy and health care utilization. Drug and Alcohol Dependence, 112, 178-193. doi:10.1016/j.drugalcdep.2010.06.014.

Azar, P., Wood, E., Nguyen, P., Luma, M., Montaner, J., Kerr, T. y Milloy, M.J. (2015). Drug use patterns associated with risk of non-adherence to antiretroviral therapy among HIV-positive illicit drug users in a Canadian setting: a longitudinal analysis. BMC Infectious Diseases, 15, 193. doi:10.1186/s12879-015-0913-0.

Blackstone, K., Woods, S.P., Weber, E., Grant, I. y Moore, D.J. (2013). Memory-based strategies for antiretroviral medication management: an evaluation of clinical predictors, adherence behavior awareness, and effectiveness. AIDS and Behavior, 17, 74-85. doi:10.1007/s10461012-0308-9.

Bragança, M. y Palha, A. (2011). Trastornos neurocognitivos asociados con la infección por el VIH. Actas Españolas de Psiquiatría, 39, 374-383. 
Cysique, L.A. y Brew, B.J. (2009), Neuropsychological functioning and antiretroviral treatment in HIV/AIDS: A Review. Neuropsychology Review, 19, 169-185. doi:10.1007/ s11065-009-9092-3.

Cohn, S.E., Jiang, H., McCutchan, J.A., Koletar, S.L., Murphy, R.L., Robertson, K.R., ... Williams, P.L. (2011). Association of ongoing drug and alcohol use with nonadherence to antiretroviral therapy and higher risk of AIDS and death: results from ACTG 362. AIDS Care, 23, 775-785. doi:10.1080/09540121.2010.525617.

Fauci, A.S. y Lane, H.C. (2005). Enfermedad por el virus de la inmunodeficiencia humana: SIDA y procesos relacionados. En: D.L. Kasper (Ed.), Harrison principios de medicina interna vol. I (pp. 1189-1265). México, D.F., México: McGraw-Hill.

Gonzalez, A., Barinas, J. y O'Cleirigh, C. (2011). Substance use: impact on adherence and HIV medical treatment. Current HIV/AIDS Reports, 8, 223-234. doi:10.1007/ s11904-011-0093-5.

González-Álvarez, S., Madoz-Gúrpide, A., Parro-Torres, C., Hernández-Huerta, D. y Ochoa Mangado, E. (2019). Relación entre la adherencia al tratamiento antirretroviral en pacientes VIH+ y el consumo de alcohol, asociado o no al uso de otras sustancias. Adicciones, 31, 8-17. doi:10.20882/adicciones.916.

Grupo de expertos del Grupo de Estudio de Sida y de la Secretaría del Plan Nacional sobre el Sida. (2014). Documento de consenso sobre el manejo clínico de los trastornos neurocognitivos asociados a la infección por el virus de la inmunodeficiencia humana (enero 2013). Enfermedades Infecciosas y Microbiología Clínica, 32, 37-47. doi:10.1016/j.eimc.2013.03.005.

Henegar, C.E., Westreich, D., Maskew, M., Brookhart, M.A., Miller, W.C., Majuba, P. y Van Rie, A. (2015). Comparison of pharmacy-based measures of adherence to antiretroviral therapy as predictors of virological failure. AIDS and Behavior, 19, 612-618. doi:10.1007/s10461-014-0953-2.

Hinkin, C.H., Castellon, S.A., Durvasula, R.S., Hardy, D.J., Lam, M.N., Mason, K.I., ... Stefaniak, M. (2002). Medication adherence among HIV+ adults: Effects of cognitive dysfunction and regimen complexity. Neurology, 59, 1944-1950.

Hinkin, C.H., Hardy, D.J., Mason, K.I., Castellon, S.A., Durvasula, R.S., Lam, M.N. y Stefaniaka, M. (2004). Medication adherence in HIV-infected adults: effect of patient age, cognitive status, and substance abuse. AIDS, 18, S19-S25.

Hinkin, C.H., Barclay, T.R., Castellon, S.A., Levine, A.J., Durvasula, R.S., Marion, S.D., ... Longshore, D. (2007). Drug use and medication adherence among HIV-1 infected individuals. AIDS and Behavior, 11, 185-194. doi:10.1007/s10461-006-9152-0.

Hutton, H.E. y Treisman, G. (2008). The role of personality in HIV risk behaviors: implications for treatment. En:
M.A. Cohen y J.M. Gorman (Ed.), Comprehensive textbook of AIDS psychiatry (pp. 141-149). New York, United States: Oxford University Press.

Ingersoll, K. (2004), The impact of psychiatric symptoms, drug use, and medication regimen on nonadherence to HIV treatment. AIDS Care, 16, 199-211. doi:10.1080/095 40120410001641048.

Kalichman, S.C., Kalichman, M.O., Cherry, C., Hoyt, G., Washington, C., Grebler, T., ... Merely, C. (2015). Intentional medication nonadherence because of interactive toxicity beliefs among HIV-positive active drug users. Journal of Acquired Immune Deficiency Syndromes, 70, 503509. doi:10.1097/QAI.0000000000000776.

Knobel, H., Alonso, J., Casado, J.L., Collazos, J., González, J., Ruiz, I., ... Ocampo, A. (2002). Validation of a simplified medication adherence questionnaire in a large cohort of HIV-infected patients: the GEEMA Study. AIDS, 16, 605-613.

Lovejoy, T.I y Suhr, J.A. (2009). The relationship between neuropsychological functioning and HAART adherence in HIV-positive adults: a systematic review. Journal of Behavioral Medicine, 32, 389-405. doi:10.1007/s10865-0099212-9.

Malta, M., Strathdee, S.A., Magnanini, M.M.F. y Bastos, F.I. (2008). Adheence to antiretroviral therapy for human immunodeficiency virus/acquired immune deficiency syndrome among drug users: a systematic review. Addiction, 103, 1242-1257. doi:10.1111/j.13600443.2008.02269.x.

Martínez-Luna, N., Rodriguez-Cintas, L., Esojo, A., Palma-Álvarez, R.F., Robles-Martínez, M., Lara Grau-López, L., ... Roncero, C. (2018). Uso de reducción de daños y gravedad psicopatológica y médica en pacientes en programa de mantenimiento con metadona. Adicciones, 30, 197-207. doi:10.20882/adicciones.897.

Meade, C.S., Conn, N.A., Skalski, L.M. y Safren, S.A. (2011). Neurocognitive impairment and medication adherence in HIV patients with and without cocaine dependence. Journal of Behavioral Medicine, 34, 128-138. doi:10.1007/ s10865-010-9293-5.

Moore, D.J., Blackstone, K., Woods, S.P., Ellis, R.J., Atkinson, J.H., Heaton, R.K. y Grant, I. (2012). Methamphetamine use and neuropsychiatric factors are associated with antiretroviral non-adherence. AIDS Care, 24, 15041513. doi:10.1080/09540121.2012.672718.

Muñoz-Moreno, J.A., Pérez-Álvarez, N., Muñoz-Murillo, A., Prats, A., Garolera, M., Jurado, M.À., ... Clotet, B. (2014). Classification models for neurocognitive impairment in HIV infection based on demographic and clinical variables. PLoS One, 9, e107625. doi:10.1371/journal. pone.0107625.

Nachega, J.B., Parienti, J.J., Uthman, O.A., Gross, R., Dowdy, D.W., Sax, P.E., ... Giordano, T.P. (2014). Lower pill burden and once-daily antiretroviral treatment re- 
gimens for HIV infection: A meta-analysis of randomized controlled trials. Clinical Infectious Diseases, 58, 12971307. doi:10.1093/cid/ciu046.

Organización Mundial de la Salud. (1992). CIE-10: Décima revisión de la clasificación internacional de enfermedades. Trastornos mentales y del comportamiento. Madrid, España: Meditor.

Ortego, C., Huedo-Medina, T.B., Vejo, J. y Llorca, F.J. (2011). Adherence to highly active antiretroviral therapy in Spain: A meta-analysis. Gaceta Sanitaria, 25, 282328. doi:10.1016/j.gaceta.2010.10.016.

Oosterman, J.M., Wijers, M. y Kessels, R.P. (2013). Planning or something else? Examining neuropsychological predictors of Zoo Map performance. Applied Neuropsychology: Adult, 20, 103-109.

Parsons, J.T., Starks, T.J., Millar, B.M., Boonrai, K. y Marcotte, D. (2014). Patterns of substance use among HIV-positive adults over 50: implications for treatment and medication adherence. Drug and Alcohol Dependence, 139, 33-40. doi:10.1016/j.drugalcdep.2014.02.704.

Paterson, D.L., Swindells, S., Mohr, J., Brester, M., Vergis, E.N., Squier, C., ... Singh, N. (2000). Adherence to protease inhibitor therapy and outcomes in patients with HIV infection. Annals of Internal Medicine, 133, 21-30.

Pedrero-Pérez, E.J. y Grupo MethaQoL. (2017). Dosis de metadona y su relación con calidad de vida, satisfacción, psicopatología, rendimiento cognitivo y consumo adicional de sustancias no prescritas. Adicciones, 29, 37-54. doi:10.20882/adicciones.831.

Peltzer, K. y Pengpid, S. (2013). Socioeconomic factors in adherence to HIV therapy in low- and middle-income countries. Journal of Health, Population and Nutrition, 31, 150-170.

Rosen, M.I., Black, A.C., Arnsten, J.H., Goggin, K., Remien, R.H., Simoni, J.M., ... Liu, H. (2013). Association between use of specific drugs and antiretroviral adherence: findings from MACH 14. AIDS and Behavior, 17, 142147. doi:10.1007/s10461-011-0124-7.

Sánchez-Cubillo, I., Periáñez, J.A., Adrover-Roig, D., Rodríguez-Sánchez, J.M., Ríos-Lago, M., Tirapu, J. y Barceló, E. (2009). Construct validity of the Trail Making Test: role of task-switching, working memory, inhibition/ interference control, and visuomotor abilities. Journal of the International Neuropsychology Society, 15, 438-450. doi:10.1017/S1355617709090626.

Shuper, P.A., Joharchi, N., Irving, H., Fletcher, D., Kovacs, C., Loutfy, M., ... Rehm, J. (2016). Differential predictors of ART adherence among HIV-monoinfected versus HIV/HCV coinfected individuals. AIDS Care. 28, 954962. doi:10.1080/09540121.2016.1158396.

Slawson, G., Milloy, M.J., Balneaves, L., Simo, A., Guillemi, S., Hogg, R., ... Kerr, T. (2015). High intensity cannabis use and adherence to antiretroviral therapy among peo- ple who use illicit drugs in a Canadian setting. AIDS and Behavior, 19, 120-127. doi:10.1007/s10461-014-0847-3.

Solomon, T.M. y Halkitis, P.N. (2008). Cognitive executive functioning in relation to HIV medication adherence among gay, bisexual, and other men who have sex with men. AIDS and Behavior, 12, 68-77. doi:10.1007/s10461007-9273-0.

Thaler, N.S., Sayegh, P., Kim, M.S., Castellon, S.A. y Hinkin, C.H. (2015). Interactive effects of neurocognitive impairment and substance use on antiretroviral non-adherence in HIV disease. Archives of Clinical Neuropsychology, 30, 114-121. doi:10.1093/arclin/acu092.

Tran, B.X., Nguyen, L.T., Nguyen, N.H., Hoang, Q.V. y Hwang, J. (2013). Determinants of antiretroviral treatment adherence among HIV/AIDS patients: a multisite study. Global Health Action, 6, 19570. doi:10.3402/gha. v6i0.19570.

Tripathi, A., Youmans, E., Gibson, J.J. y Duffus, W.A. (2011). The impact of retention in early HIV medical care on viro-immunological parameters and survival: a statewide study. AIDS Research and Human Retroviruses, 27, 751-758. doi:10.1089/AID.2010.0268.

Tsuyuki, K. y Surratt, H.L. (2015). Antiretroviral drug diversion links social vulnerability to poor medication adherence in substance abusing populations. AIDS and Behavior, 19, 869-881. doi:10.1007/s10461-014-0969-7.

Vázquez-Justo, E., Vergara-Moragues, E., Piñón-Blanco, A., Guillén-Gestoso, C. y Pérez-García, M. (2016). Neuropsychological functioning in methadone maintenance patients with HIV. Revista Latinoamericana de Psicología, 48, 147-158. doi:10.1016/j.rlp.2015.06.008.

Viswanathan, S., Justice, A.C., Alexander, G.C., Brown, T.T., Gandhi, N.R., McNicholl, I.R., ... Jacobson, L.P. (2015). Adherence and HIV RNA suppression in the current era of highly active antiretroviral therapy. Journal of Acquired Immune Deficiency Syndromes, 69, 493-498. doi:10.1016/j. rlp.2015.06.008.

Wagner G.J. (2002). Predictors of antiretroviral adherence as measured by self-report, electronic monitoring, and medication diaries. AIDS Patient Care and STDs, 16, 599608.

Waldrop-Valverde, D., Ownby, R.L., Wilkie, F.L., Mack, A. Kumar, M. y Metsch, L. (2006). Neurocognitive aspects of medication adherence in HIV-positive injecting drug users. AIDS and Behavior, 10, 287-297. doi:10.1007/ s10461-005-9062-6. 